\title{
Theory of reactions at electrified interfaces
}

\author{
$\underline{\text { Jessica Lück }}^{\star a b}$ and Arnulf Latz $^{\text {abc }}$
}

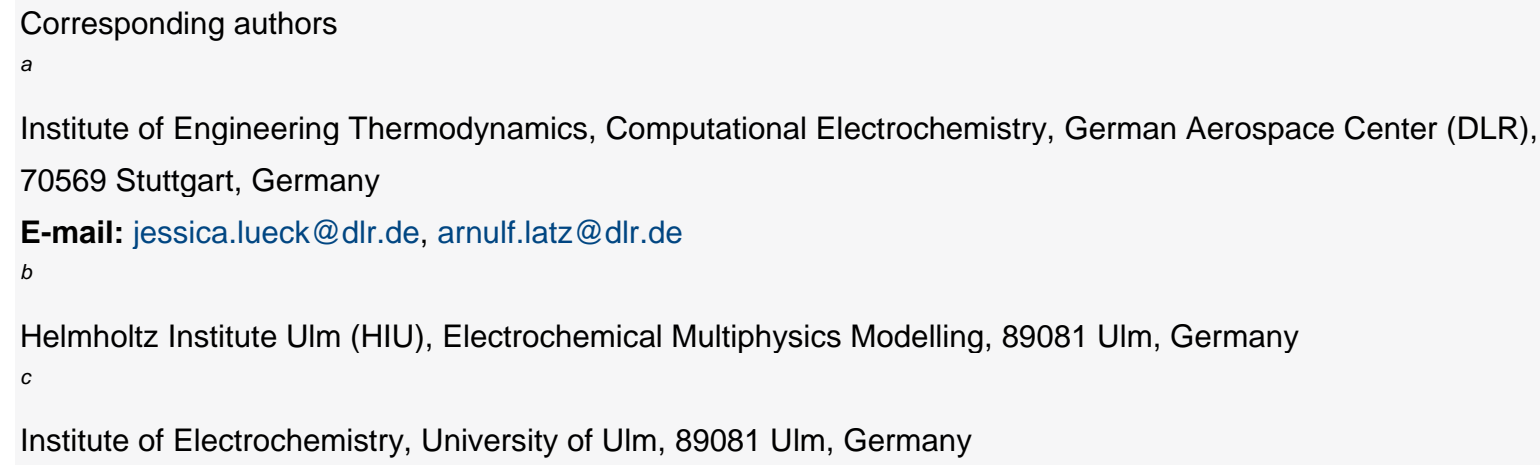

Phys. Chem. Chem. Phys., 2016,18, 17799-17804

DOI: $10.1039 / \mathrm{C} 6 \mathrm{CP} 02681 \mathrm{H}$

Received 21 Apr 2016, Accepted 18 May 2016

First published online 18 May 2016

Interfacial reaction and transport processes are a decisive factor for the overall performance of electrochemical systems. However, existing models rely on phenomenological descriptions of charged interfaces, which yields no deeper insights. We present a generic theory to describe charge and electron transfer reactions at charged interfaces, which is applicable to different electrochemical systems, like fuel cells or batteries with liquid or solid electrolytes. In the present work, our general theory is adopted to the electrochemical double layer at the interface between a solid electrode and a liquid electrolyte. The model allows to describe the intercalation reaction in Li-ion insertion batteries as a two-step process, consisting of a first desolvation and adsorption and a second actual insertion step. It becomes apparent that a charging of the double layer acts as the necessary driving force for the charge transfer across the interface. 